\title{
On the acquisition of Greek free choice items
}

Evangelia Vlachou ${ }^{1}$, Dimitrios Kotopoulis ${ }^{2}$, Spyridoula Varlokosta ${ }^{2}$

${ }^{1}$ Department of French, National and Kapodistrian University of Athens, Greece ${ }^{2}$ Department of Linguistics, National and Kapodistrian University of Athens, Greece https://doi.org/10.36505/ExLing-2020/11/0053/000468

\begin{abstract}
Children acquire quite late scalar implicatures associated with quantification and have the tendency to interpret existential quantifiers as universals (e.g., Smith, 1980; Noveck, 2001; Papafragou and Musolino, 2003). Free choice Items (FCIS) are also associated with scalar implicatures depending on whether they are full set or subset FCIs (e.g., Vlachou 2012, 2020). This paper presents experimental results showing that 9-, 10- and 11 -year-old children and adults perform better on full set than on subset FCIs. It is argued that adults perform better than children in sentences with subset FCIs as the "not-all" pragmatic inference is acquired late. Difficulties in sentences with subset FCIs in adults are due to absence of domain alternatives.
\end{abstract}

Keywords: free choice items, scalar implicatures, quantification, alternatives, semantics

\section{Introduction}

The literature on the acquisition of quantifiers (e.g., Noveck, 2001; Papafragou and Musolino, 2003; Smith, 1980) has put forward the hypothesis that children acquire quite late scalar implicatures associated with quantification and have the tendency to interpret existential quantifiers as universals: the "not-all" meaning component of existential quantifiers is acquired only at a later stage.

The close relation between quantification and Free Choice Indefinites (FCIs) such as any has been extensively documented (see Dayal, 1998 and Kadmon and Landman, 1993 inter alia).

(1) a. Any student can solve this problem.

b. All students can solve this problem.

(2) a. I did not talk to just any student.

b. I did not talk with some student or other.

Vlachou $(2012,2020)$ argued that, the core semantic property that defines FCIs as a class and distinguishes them from other lexical items is that they have descriptive contents that, except for referring to alternatives, bring information on these alternatives. They form two different interpretational categories: full set, associated with universal quantification over alternatives (an "all" meaning) and subset FCIs, associated with existential quantification over alternatives (a "not-all" meaning). Full set FCIs (opjosdhipote (o-dhipote, hereafter) require that all

ExLing 2020: Proceedings of $11^{\text {th }}$ International Conference of Experimental Linguistics, 12-14 October 2020, Athens, Greece 
alternatives, without exception, are considered. Subset FCIs (subset 1: enas/o opjosdhipote (enas/o o-dhipote, hereafter); subset 2: opjos opjos (o- o-, hereafter)) restrict the domain of quantification of the alternatives to one of its subsets.

The difference in the acquisition of universal and existential quantifiers makes one predict that the acquisition of full set and subset FCIs differs, as well. Moreover, recent accounts, as in Tieu et al. (2015), have shown that the acquisition of scalar implicatures becomes harder when relevant alternatives are not provided by the context. To test both predictions, we set up an experiment to investigate the tendencies in the acquisition of Greek FCIs. Methodology

\section{Participants}

The sample consisted of 154 participants: 120 children - 40 9-year-old attending 4th grade (age range: 9;6 - 10;5; mean age: 9;11), 40 10-year-old attending 5 th grade (age range: 10;6 - 11;5; mean age: 10;11), and 4011 -year-old attending 6 th grade(age range: $11 ; 6$ - 12;5; mean age: 11;11) - and a control group of 34 adults. All participants were monolingual native speakers of Greek.

\section{Materials}

We developed two written tasks, assessing production (Task $A$ ) and comprehension (Task B) of FCIs. Task $A$ was a sentence completion task that contained 60 sentences with gaps: 30 sentences had a full set FCI as target item (3) and 30 sentences a subset FCI (4). Task B contained 40 pairs of sentences. In each pair, the two sentences were identical, except for the FCI they contained; one always had a full set FCI (5a), while the other a subset FCI (5b).

(3) Tha eprepe na kseris oti egho s'aghapo perisotero apo opjondhipote alo. 'You should know that I love you more than anybody else.'

(4) Egho dhen ime (enas/o opjosdhipote / opjos ki opjos). Ime o kaliteros su filos.

'I'm not just anyone. I am your best friend.'

(5) a. Opjosdipote mathitis echi tsanda. (target) 'Any pupil has a schoolbag.'

b. \#?Enas opjosdhipote mathitis echi tsanda.

Sentences in both tasks were fairly short and deprived of (long) pragmatic context in order to examine how participants produce and comprehend "out of the blue" sentences with FCIs. If not, our experiment would corroborate the validity of the hypothesis that pragmatic context is relevant to the understanding of FCIs. 


\section{Results}

Significant differences were observed among the four age groups in both tasks regarding the mean scores of successful performance (see Table 1). As expected, fourth graders scored lower than the other three groups, while adults exhibited the highest mean scores. In Task A, a significant difference arose between the performance of fourth graders and that of all other groups, while in Task B, the adults' performance differed significantly from that of all the other groups (Table 2).

Table 1. Mean scores and statistics for the four age groups in the two tasks.

\begin{tabular}{|l|l|l|}
\hline Group & Task A Mean (Range) & Task B Mean (Range) \\
\hline $4^{\text {th }}$ grade & $34.93(8-54)$ & $24.68(16-33)$ \\
\hline $5^{\text {th }}$ grade & $41.25(21-55)$ & $25.7(14-39)$ \\
\hline $6^{\text {th }}$ grade & $41.8(22-56)$ & $27.43(18-35)$ \\
\hline adults & $44.41(30-52)$ & $31.65(24-38)$ \\
\hline Kruskal-Wallis & $\chi^{2}=23.51, \mathrm{df}=3, \mathrm{p}=.000$ & $\chi^{2}=49.69, \mathrm{df}=3, \mathrm{p}=.000$ \\
\hline
\end{tabular}

Table 2. Statistics for pair-wise between-groups comparisons.

\begin{tabular}{|c|c|c|c|c|}
\hline & \multicolumn{2}{|l|}{ TASK A } & \multicolumn{2}{|l|}{ TASK B } \\
\hline Comparisons & Test Value (U) & $\mathrm{p}$-value & Test Value $(\mathrm{U})$ & $p$-value \\
\hline $4^{\text {th }}$ vs. $5^{\text {th }}$ grade & 467 & .001 & 703.5 & .35 \\
\hline $4^{\text {th }}$ vs. $6^{\text {th }}$ grade & 461 & .001 & 582 & .03 \\
\hline $4^{\text {th }}$ vs. adults & 264 & .000 & 98.5 & .000 \\
\hline $5^{\text {th }}$ vs. $6^{\text {th }}$ grade & 759 & .69 & 681.5 & .25 \\
\hline $5^{\text {th }}$ vs. adults & 498 & .04 & 156.5 & .000 \\
\hline $6^{\text {th }}$ vs. adults & 562.5 & .2 & 236 & .000 \\
\hline
\end{tabular}

In both tasks, the overall difference in correct answers between full set and subset FCIs yielded a significant result (Task A: $\mathrm{Z}=-9.051, \mathrm{p}=.000$; Task B: $\mathrm{Z}$ $=-10.448, p=.000)$. The subsequent testing of differences between the two types of FCIs within each group for each task showed that all four groups performed better in full set than in subset FCIs (Table 3).

Table 3. Means and statistics for full set vs. subset comparisons per group.

\begin{tabular}{|c|c|c|c|c|c|c|}
\hline & \multicolumn{3}{|c|}{ TASK A } & \multicolumn{3}{|l|}{ TASK B } \\
\hline & \multicolumn{2}{|c|}{ Means } & \multirow[t]{2}{*}{ Statistics $(Z ; p)$} & \multicolumn{2}{|l|}{ Means } & \multirow[t]{2}{*}{ Statistics $(Z ; p)$} \\
\hline Group & Full Set & Subset & & Full Set & Subset & \\
\hline $4^{\text {th }}$ grade & 25.1 & 9.83 & $-5.159 ; .000$ & 14.97 & 7.68 & $-5.129 ; .000$ \\
\hline $5^{\text {th }}$ grade & 26.3 & 14.95 & $-5.352 ; .000$ & 15.85 & 2.06 & $-5.521 ; .000$ \\
\hline $6^{\text {th }}$ grade & 25.22 & 16.57 & $-4.189 ; .000$ & 15.15 & 9.65 & $-5.283 ; .000$ \\
\hline Adults & 24.94 & 19.47 & $-2.608 ; .009$ & 17.50 & 11.79 & $-5.046 ; .000$ \\
\hline
\end{tabular}




\section{Discussion and conclusions}

Our prediction that full set FCIs are acquired earlier than subset FCIs was born out by the results of this study. Our experiment revealed that 9-, 10- and 11year-old children have a clear tendency of using and comprehending sentences with full set FCIs over sentences with subset FCIs. Moreover, the acquisition of sentences with subset FCIs becomes better as age progresses but is not adequate yet at the age of 11. Subset and full set FCIs follow different acquisitional paths, as they have different semantic features. Subset FCIs are associated with a "not-all" implicature, known to cause problems to children. Full set FCIs, on the other hand, are the default option for 9-, 10- and 11-yearold children, as they are associated with universal quantification over alternatives, known not to cause any problem in the acquisition of quantification.

Our experiment leads us to another interesting finding that puts our study in line with studies that have detected difficulties in the comprehension and production of sentences with "not-all" implicatures in adults too (e.g., Tieu et al. 2015). Even adults do not perform well on scalar implicatures when they are unable to retrieve the alternatives in question. Once alternatives are provided, they both comprehend and produce sentences with scalar items. Returning to our experiment, data contained fairly short sentences deprived of any pragmatic context or any picture that would enable participants to retrieve the alternatives in question. Thus, our results serve as further evidence for the validity of the hypothesis that once alternatives are not provided by the context, even adult participants have difficulties computing sentences with FCIs.

\section{References}

Dayal, V. 1998. Any as inherently modal. Linguistics and philosophy 21, 433-476.

Kadmon, N., Landman, K. 1993. Any. Linguistics and philosophy 16, 353-422.

Noveck, I. 2001. When children are more logical than adults: experimental investigations of scalar implicature. Cognition 78, 165-188.

Papafragou, A., Musolino, J. 2003. Scalar implicatures: experiments at the semanticspragmatics interface. Cognition, 86(3), 253-282.

Smith, C. 1980. Quantifiers and question answering in young children. Linguistic investigations. Journal of experimental child psychology 40, 191-205.

Tieu, L., Romoli, J., Zhou, P., Crain, St.. 2016. Children's knowledge of free choice inferences and scalar implicatures. Journal of semantics 33(2), 269-298.

Vlachou, E. 2012. Delimiting the class of free choice items in a comparative perspective: evidence from the database of the French and Greek free choice items. Lingua 122(14), 1523-1568.

Vlachou, E. 2020. Indéfinis et implicatures: le cas de n’ importe qu- et un $N$ quelconque. In C. Beyssade (ed.) Implicature. ISTE. 